\title{
SIMULATION ANALYSIS FOR INTERACTIVE RETRIEVAL OF SPOKEN DOCUMENTS WITH KEY TERMS RANKED BY REINFORCEMENT LEARNING
}

\author{
Yi-cheng Pan and Lin-shan Lee \\ Graduate Institute of Computer Science and Information Engineering, National Taiwan University \\ Taipei, Taiwan, Republic of China \\ thomas02@is.sinica.edu.tw
}

\begin{abstract}
Unlike written documents, spoken documents are difficult to display on the screen; it is also difficult for users to browse these documents during retrieval. It has been proposed recently to use interactive multi-modal dialogues to help the user navigate through a spoken document archive to retrieve the desired documents. This interaction is based on a topic hierarchy constructed by the key terms extracted from the retrieved spoken documents. In this paper, the efficiency of the user interaction in such a system is further improved by a key term ranking algorithm using Reinforcement Learning with simulated users. Extensive simulation analysis was performed, and significant improvements in retrieval efficiency were observed. These improvements appeared that the proposed method is relatively robust to speech recognition errors.
\end{abstract}

\section{INTRODUCTION}

In a recent paper, we proposed using multi-modal dialogues to help the user "navigate" across a spoken document archive to retrieve the desired documents [1]. For a given user query, the retrieval system produces a topic hierarchy constructed from the retrieved spoken documents to be shown on the screen of the hand-held devices. The user can then expand his query easily by choosing the key terms [2] within the topic hierarchy by a simple click or an additional spoken query to specify more clearly what he is looking for. With a few dialogue turns, the small set of spoken documents desired by the user can be found by a more specific query expanded during the dialogue process.

Given the user's initial query, however, a large number of key terms are usually extracted and the constructed topic hierarchy is very often too large to be shown on the screen of a hand-held device. In this paper, we further propose a new ranking algorithm for the key terms based on Reinforcement Learning [3, 4, 5, 6] considering both the semantic structure of the document archive and the information needs of the user. Extensive simulation analysis showed that training the ranking algorithm directly based on the search efficiency and user satisfaction offers improved retrieval efficiency over conventional term ranking methods used in the area of information retrieval. The proposed ranking algorithm is also shown to be robust against recognition errors.

\section{KEY TERM RANKING BY REINFORCEMENT LEARNING WITH SIMULATED USERS}

In the proposed approach for spoken document retrieval/navigation (SDR), the input user query is usually very short and the retrieved documents very numerous. A large number of key terms are usually

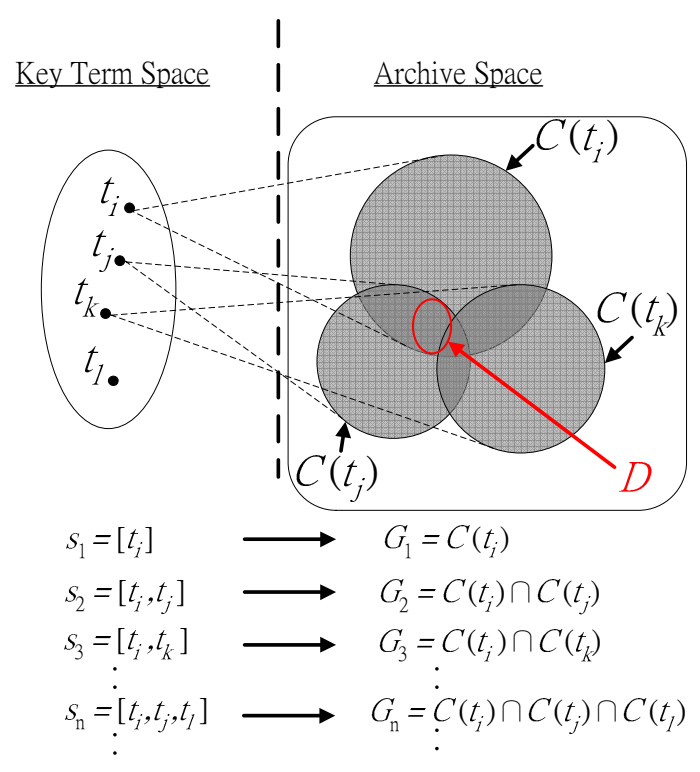

Fig. 1. Key term space and archive space.

extracted and the constructed topic hierarchy hence tends to be too large. It is therefore crucial to have a good strategy for ranking all of these key terms and for moving these important key terms towards the top of each layer of the hierarchy, so as to make it easier for the user to choose, and thus to produce efficient interaction between the user and the system. Such a term ranking strategy has to achieve a good balance between two mutually contradicting factors of the key terms - i.e., coverage (more documents can be retrieved with the key term) and discriminating ability (the key term indicates a more specific topic against other irrelevant documents) — by reflecting the semantic structure of the document archive. The approach proposed here for the above purpose is based on the well known concept of Reinforcement Learning [3, 4, 5, 6]. In this approach, the training data used are simply those generated by simulated users.

\subsection{Key term space and archive space}

As shown in the left half of Fig. 1, all the key terms $t_{i}, t_{j}, t_{k}, t_{l}$, ... for a given document archive form a key term space. Every key term $t_{i}$ can be mapped to a class of documents $C\left(t_{i}\right)$ which can be retrieved by the term $t_{i}$ with a given retrieval approach in the archive space as shown in the right half of Fig. 1. Thus the mapping relation between the key term $t_{i}$ and the corresponding document class $C\left(t_{i}\right)$ 
is defined by the given retrieval approach, and the formulation here is equally applicable to all retrieval approaches, including the Vector Space Model, Latent Semantic Indexing (LSI) [7], or Probabilistic Latent Semantic Indexing (PLSI) [8].

The user may initiate the retrieval process by entering the first key term $t_{i}$ as the query, which is referred to as the first state $s_{1}$; the retrieved results are the group of documents $G_{1}$, or

$$
s_{1}=\left[t_{i}\right] \longrightarrow G_{1}=C\left(t_{i}\right) .
$$

When $G_{1}$ is very large, the user may further enter the next key term $t_{j}$ or $t_{k}$ to expand his query, respectively referred to as states $s_{2}$ or $s_{3}$, and the retrieved results are denoted as $G_{2}$ or $G_{3}$,

$$
\begin{aligned}
& s_{2}=\left[t_{i}, t_{j}\right] \longrightarrow G_{2}=\left[C\left(t_{i}\right)\right] \cap\left[C\left(t_{j}\right)\right], \\
& s_{3}=\left[t_{i}, t_{k}\right] \longrightarrow G_{3}=\left[C\left(t_{i}\right)\right] \cap\left[C\left(t_{k}\right)\right],
\end{aligned}
$$

and so on. This process can continue: for example, the query for state $s_{2}$ above can be further expanded by a third key term $t_{l}$ to produce another state $s_{n}$ with the retrieved results $G_{n}$,

$$
s_{n}=\left[t_{i}, t_{j}, t_{l}\right] \longrightarrow G_{n}=\left[C\left(t_{i}\right)\right] \cap\left[C\left(t_{j}\right)\right] \cap\left[C\left(t_{l}\right)\right] .
$$

\subsection{Reinforcement Learning by Simulated Users}

In the reinforcement learning algorithm, we need a huge quantity of training data, which are generated by simulated users as described below.

\subsubsection{Simulating the User's Information Needs}

Here we simulate the information needs of the user, which is a set of desired documents, $D$, in the archive space. In observing real users' information needs, we found two characteristics: 1) the documents in $D$ have similar semantics to each other, and 2) the documents in $D$ usually share common key terms. So we first cluster the whole document archive into $C$ clusters. $C$ should be chosen such that each cluster has enough documents ${ }^{1}$. Then we randomly pick one cluster and extract all of the key terms from the documents in that cluster. A key term $\bar{t}$ is then randomly chosen from the extracted key terms, and the set of documents including this key term $\bar{t}$ is denoted as $D^{\prime}$. A random number $M$ is generated as the size of the desired document set $D$. If the number of documents in $D$ ' exceeds $M$, exactly $M$ documents are randomly selected and the set of them is regarded as $D$. Otherwise, we choose another key term most similar to $\bar{t}$ but not yet chosen and add documents including this key term into $D$ ' until the number of documents in $D^{\prime}$ equals or exceeds $M$. The similarity used here is based on the probability distribution of each key term on the latent topics in the same Probabilistic Latent Semantic Analysis (PLSA) model used in the PLSI retrieval process ${ }^{2}$. For example, the key term "White House" may result in the most similar key terms "U.S.", "George Bush", "Middle East", and so on. The set of desired documents $D$ generated in this way in general satisfies the two requirements mentioned above.

\subsubsection{Simulating the Retrieval Behavior of a User}

With the desired document set $D$ generated as above for a simulated user, we then simulate the user's retrieval behavior as follows. A key term $t_{i}$ which can retrieve at least some documents in $D$ is randomly selected as the first query, or state $s_{1}$, for this simulated user, and

\footnotetext{
${ }^{1}$ In our system, $C$ was set as 12 .

${ }^{2}$ In our system, we used 350 latent topics.
}

the group of documents $G_{1}=C\left(t_{i}\right)$ is obtained as in relation (1). Very possibly $G_{1}$ is much larger than the desired document set $D$, so the simulated user may enter the next key term $t_{j}$ or $t_{k}$, and so on. In general, $t_{j}$ or $t_{k}$ can be any key terms that can retrieve at least some documents in $C\left(t_{i}\right)$. Each of these possible key terms is then simulated as $s_{2}=\left[t_{i}, t_{j}\right], s_{3}=\left[t_{i}, t_{k}\right]$, producing the retrieved results $G_{2}, G_{3}$ and so on as in relations (2)(3). This process can continue: for example, entering the third key term $t_{l}$ after $s_{2}=$ $\left[t_{i}, t_{j}\right]$ as state $s_{n}=\left[t_{i}, t_{j}, t_{l}\right]$ as in relation (4). Assume the user is satisfied when the recall rate $r$ is above a threshold $r_{0}$, i.e., $L$ out of the top $K$ retrieved documents are within the desired document set $D$, or $r=L / M>r_{0}$, where $M$ is the number of documents in $D$.

The above simulation results for a simulated user with a desired document set $D$ and an initial key term $t_{i}$ can be represented by a tree of all the states as defined in relations (1)(2)(3)(4), and a typical example of such a tree is partially shown in Fig. 2. For example, after the first key term is entered as state $s_{1}$, different choices of the second key term result in the different states $s_{2}, s_{3}$ and $s_{4}$ on the next layer, and so on. The leaf nodes of the tree represented by double circles are the final states - or those states where the user is satisfied - labeled by a score $m(\cdot)$ which is the number of key terms successively entered, or the number of steps required to arrive at the final state along the path from the root. Clearly, the smaller $m(\cdot)$ the better. After the tree is constructed, backtracking from each leaf node along a path back to the root gives a score $u$ to each intermediate state, which is the minimum score $m(\cdot)$ for all child leaf nodes of the intermediate state as below,

$$
u=\min _{i}\left[m\left(s_{i}\right)\right],
$$

where the minimization is performed over all child leaf nodes of the state. For example, in Fig. $2 u=3$ for $s_{2}$ because $m\left(s_{6}\right)=3$, but $u=4$ for $s_{5}$ because $m\left(s_{8}\right)=4$. For a given parent node, the nextlayer child node with smaller score $u$ is clearly better, because the user can be satisfied earlier by following that key term. For example, for the three states $s_{2}, s_{3}, s_{4}$ in the second layer in Fig. 2, $s_{4}$ is better than $s_{2}$, and $s_{2}$ is in turn better than $s_{3}$. Note that in order to avoid excessively lengthy path expansions in the trees we need to prune the tree branch if no documents can be retrieved at the branching state.

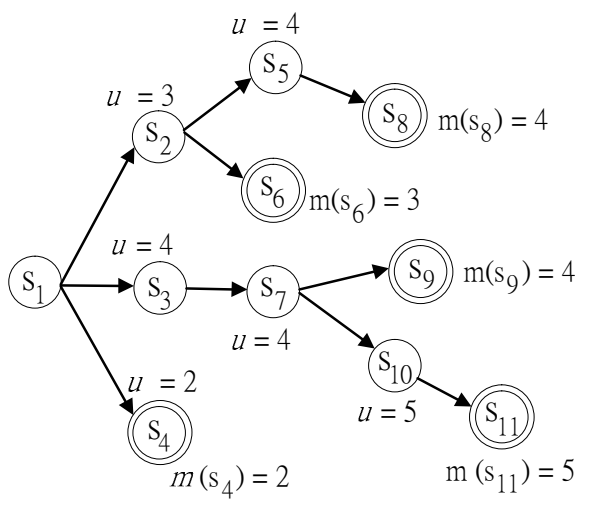

Fig. 2. A typical tree constructed for the retrieval states for a simulated user.

\subsection{The Proposed Ranking Algorithm}

It should be noted that given the documents archive and a set of key terms, the document classes $C\left(t_{i}\right)$ for all key terms $t_{i}$ are fixed, as are the intersection relationships among them such as $G_{2}, G_{3}, G_{4}$ 
in relations (2)(3)(4). As a result, when starting with a certain state $s_{1}=\left[t_{i}\right]$ as in relation (1), all the possible next key terms such as $t_{j}$, $t_{k}$ in relations (2)(3) are also fixed, as are the next layer states in the tree in Fig.2. In other words, the fundamental structure of the tree in Fig. 2 given the root $s_{1}$ is fixed, but the specific realization of the tree is dependent on the desired document set $D$; that is, different desired document sets $D$ will in effect prune the branches of the fundamental tree structure at different layers, thereby producing a different set of leaf nodes for each $D$.

As mentioned previously, every simulated user is defined by a simulated desired document set $D$ and an initial query key term $t_{i}$, followed by all possible following key terms $t_{j}, t_{k}$ and so on. The initially large number of users can be further classified into categories by the initial query key term $t_{i}$. In each category all the simulated users start with the same initial query key term $t_{i}$ and the same root state $s_{1}=\left[t_{i}\right]$, and thus have the same basic tree structure, but the different desired document sets $D$ for the different simulated users produce different leaf nodes and different tree realizations, which result in different scores as in equation (5) for each state in the basic tree structure. All different scores $u$ for the different tree realizations for a certain state $s_{j}$ in the basic tree structure can then be averaged over all of the tree realizations to give an averaged score $\bar{u}$ for the state $s_{j}$. This produces a single tree for a category of users starting with the same initial key term, $t_{i}$. This tree describes the retrieval efficiency of all of the possible expansions of the initial query, which can be seen as the "averaged tree" for an initial key term $t_{i}$

The set of all "averaged trees" as mentioned above for all initial key terms $t_{i}$ is then the optimized ranking formula. It reflects the semantic structure of the document archive, and indicates how efficient each key term is in identifying the desired documents given the previously entered queries, which constitute a key term set; that is, a state. This efficiency represents an automatic balance between two mutually contradicting factors for the key terms as mentioned previously: coverage and discriminating ability. Such a balance is obtained by averaging the retrieval processes of a large number of simulated users.

Taking into consideration the SDR problem, it can be seen that after the real user enters a query, a topic hierarchy is constructed for the many retrieved documents with nodes labeled by key terms. The many key terms on a level of the topic hierarchy are then sorted according to this ranking algorithm. In this way, the user can select the next key term from the top to expand his query and indicate his information need precisely and efficiently, even on a small screen, i.e., of a hand-held device.

\section{EXPERIMENTAL RESULTS}

An initial prototype system was successfully developed at National Taiwan University (NTU). Mandarin Chinese broadcast news segments were taken as the example spoken/multi-media documents. Here the broadcast news archive to be navigated across and retrieved from included 10,000 news stories, among which about 2000 key terms were extracted [9]. A total of 5,000,000 users were simulated in training the ranking algorithm.

We evaluated the performance of interactive retrieval in terms of task success rate and the number of key terms needed for a successful retrieval. The task is defined to be successful if the user is satisfied after entering a number of queries, as defined in Sec. 2.2.2, i.e., $L$ out of the top $K$ retrieved documents are within the desired document set $D$, and $L / M>r_{0}$, where $M$ is the number of documents in $D$ and $r_{0}$ is the desired recall rate. $M$ was taken as a random number uniformly distributed in $[5,100]$. $K$ was set to 25 and $r_{0}$ to 0.15 . In the test process, before user satisfaction the test user is requested to follow the ranking list proposed by the system to see whether the current top ranking key term is desired, or appears in at least one of the documents in his information need, $D$. If the top ranking key term is not desired, the user goes to the next and so on. Once the first desired key term is found, the user simply chooses that key term to augment his query. Another set of retrieved documents and another ranking of the respective key terms are then displayed to the user to judge the user's satisfaction or to proceed the next run.

We generated 1000 test users in the same way as we simulated the training users as presented in Secs. 2.2.1 and 2.2.2. Two graduate students of NTU's Graduate Institute of Journalism helped to perform another 50 real user tests including producing the desired document set $D$ and the entered queries. The proposed ranking algorithm is compared against two previously proposed algorithms, the $w p q$ method proposed by Robertson [10] and the tf-idf method. All three methods were applied on the same set of key terms extracted from the spoken documents.

\subsection{Average depth for the first desired key term}

This experiment is to find out how far in average percentage each user has to go down the ranked key term list to find the first desired key term for each query augmentation. The results are listed in Table 1. This experiment is to see how good a ranking approach can filter out non-relevant terms and rank the relevant ones as high as possible. It can be found that the proposed approach is slightly better than the $w p q$ method, while the $t f-i d f$ method is much worse.

\begin{tabular}{|l|c|c|c|}
\hline & $\begin{array}{l}\text { Proposed } \\
\text { approach }\end{array}$ & $\begin{array}{l}w p q \\
\text { method }\end{array}$ & $\begin{array}{l}t f-i d f \\
\text { method }\end{array}$ \\
\hline $\begin{array}{l}\text { average depth for the } \\
\text { first desired key term }\end{array}$ & $28 \%$ & $30 \%$ & $56 \%$ \\
\hline
\end{tabular}

Table 1. The average normalized depth that the test user needs to go down to find the first desired key term.

\subsection{Task success rate and average number of key terms needed}

The complete simulation results tested on 1000 simulated testing users are listed in Table 2. In the upper part (I) including part (A), the speech recognition for the queries was assumed to be $100 \%$ correct, while the character recognition accuracies were assumed to degrade to lower numbers in the lower part (II) including parts (B)(C)(D). In cases $(B)(C)(D)$, we simulated the recognition errors for each query both in the testing and the training set by generating feature vectors according to the Hidden Markov Models with increased Gaussian mixture variances. During training, simulated queries for each initial query were generated with random errors, which in turn generated trees slightly different from that for the initial query and so on. Two performance measures were used here, the task success rate $R_{\text {success }}$ and the average number of key terms needed $N_{\text {keyterm }}$. For both measures three cases of archives to be retrieved are tested. In case (1) all the 10,000 news stories are in text form without recognition errors. In case (2) 1700 out of the 10,000 news stories are spoken with character recognition accuracy of $77 \%$. In case (3) 1000 out of the 10,000 are spoken with character accuracy of $71 \%$. The recognition errors were generated in exactly the same way as those in the queries mentioned above.

From part (A) and case (1) of Table 2, we see that with the proposed ranking algorithm, the best task success rate of $89.2 \%$ can 
be achieved, as compared to the $78.6 \%$ and $33.1 \%$ for $w p q$ method and $t f-i d f$ methods respectively. Also, the average number of key terms needed for successful trials is as low as 2.13 for the proposed ranking algorithm, as compared to 3.08 and 3.46 for $w p q$ and tf-idf methods. Similar situations can be observed in cases (2) and (3). It can be found that the results are reasonably robust with respect to recognition errors.

Fig. 3 (a) shows the detailed numbers of failure trials and successful trials completed in different number of key terms out of the 1000 simulated testing users for part (A) and case (3) of Table 2. It was found that with the tf-idf method, 746 out of the 1000 trials failed; all successful trials were finished within 7 turns. Much better performance was obtained for the $w p q$ method. However, when the proposed ranking algorithm was used, only 120 trials failed, and all trials were completed within 4 turns, a result significantly better even than the $w p q$ method. Consistent situations can be observed in all other parts and cases of Table 2. For example, in part (D) and case (2), the proposed approach still achieved a success rate of $74.9 \%$, as compared to the serious degradations in the other approaches, because in those cases the recognition errors simply led to totally irrelevant key terms. Similar plots for the detailed numbers in part (D) and case (2) can be seen in Fig. 3 (b).

In Fig. 4 we plot the task success rate and average number of key terms needed in successful trials for the three ranking methods in cases (1) and (2) as functions of the recognition accuracies for the queries. It can be found that the performance of the proposed ranking algorithm is quite robust with respect to recognition errors.

\begin{tabular}{|c|c|c|c|c|c|c|c|c|}
\hline \multirow{5}{*}{ (I) } & \multirow{2}{*}{$\begin{array}{l}A C C \text { in } \\
\text { queries }\end{array}$} & \multirow[t]{2}{*}{ Algorithms } & \multicolumn{3}{|c|}{$R_{\text {success }}$} & \multicolumn{3}{|c|}{$N_{\text {key term }}$} \\
\hline & & & (1) & (2) & (3) & (1) & (2) & (3) \\
\hline & \multirow{3}{*}{$\begin{array}{l}\text { (A) } \\
100 \%\end{array}$} & Proposed & 89.2 & 89.0 & 88.0 & 2.13 & 2.16 & 2.30 \\
\hline & & $w p q$ & 78.6 & 77.4 & 73.8 & 3.08 & 3.21 & 3.45 \\
\hline & & $t f-i d f$ & 33.1 & 30.4 & 25.4 & 3.46 & 3.77 & 4.03 \\
\hline \multirow{9}{*}{ (II) } & \multirow{3}{*}{$\begin{array}{l}\text { (B) } \\
92 \%\end{array}$} & Proposed & 89.1 & 87.0 & 82.0 & 2.21 & 2.29 & 2.55 \\
\hline & & $w p q$ & 75.2 & 72.1 & 64.3 & 4.33 & 4.53 & 4.66 \\
\hline & & $t f-i d f$ & 30.1 & 22.1 & 16.1 & 5.78 & 5.89 & 6.01 \\
\hline & \multirow{3}{*}{$\begin{array}{l}\text { (C) } \\
88 \%\end{array}$} & Proposed & 86.0 & 82.0 & 75.9 & 2.33 & 2.49 & 2.68 \\
\hline & & $w p q$ & 70.5 & 64.5 & 53.5 & 4.89 & 5.12 & 5.67 \\
\hline & & $t f-i d f$ & 21.0 & 10.2 & 8.1 & 6.01 & 6.12 & 6.15 \\
\hline & \multirow{3}{*}{$\begin{array}{l}\text { (D) } \\
74 \%\end{array}$} & Proposed & 80.1 & 74.9 & 65.6 & 3.34 & 3.58 & 3.92 \\
\hline & & $w p q$ & 55.2 & 45.2 & 32.7 & 5.23 & 5.44 & 5.99 \\
\hline & & $t f-i d f$ & 11.2 & 7.5 & 3.3 & 6.31 & 6.43 & 6.51 \\
\hline
\end{tabular}

Table 2. The results for the proposed ranking algorithm compared with the $w p q$ and $t f-i d f$ methods, without and with recognition errors in queries, tested by 1000 simulated testing users. $R_{\text {success }}$ is the task success rate in percentage and $N_{\text {key }}$ term the average number of key terms needed in a successful retrieval.

\subsection{Results for the Ranking Algorithm by Real Users}

Here we performed 50 tests handcrafted by two graduate students of NTU's Graduate Institute of Journalism with their desired document sets $D$ and their initial queries, and the results are listed in Table 3. It can be found that with human testers, a similar situation to that mentioned above can be observed.

\begin{tabular}{|c|c|c|}
\hline Experiments & $R_{\text {success }}$ & $N_{\text {key } \text { term }}$ \\
\hline Proposed ranking algorithms & $81.0 \%$ & 2.62 \\
\hline$w p q$ method & $79.0 \%$ & 3.10 \\
\hline$t f-i d f$ method & $28.0 \%$ & 3.54 \\
\hline
\end{tabular}

Table 3. Experimental results for the different ranking algorithms, tested by human users.
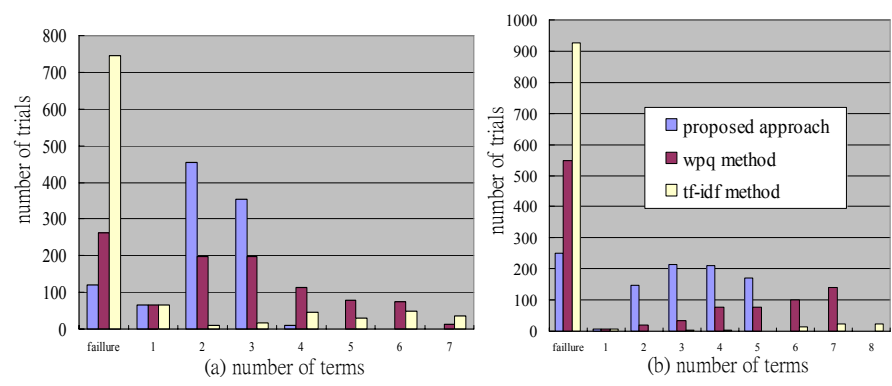

Fig. 3. Number of failure trials and successful trials completed in different number of tkey terms for the proposed ranking algorithm compared to the $w p q$ and $t f-i d f$ methods. (a) for part (A) and case (3) and (b) for part (D) and case (2) in Table 2
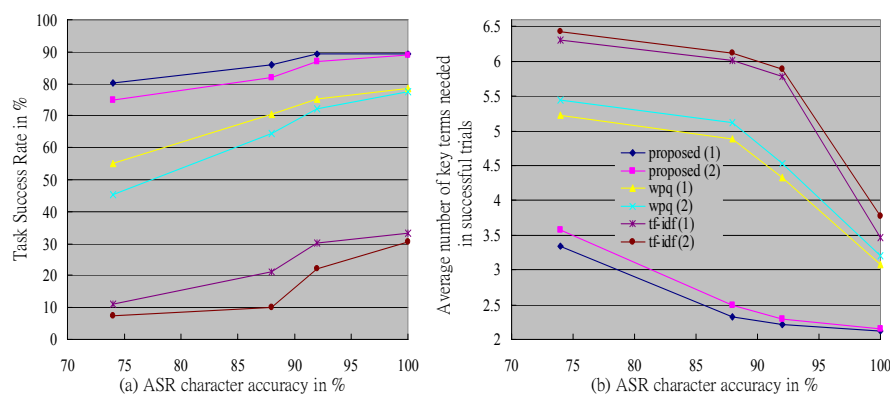

Fig. 4. (a) the task success rates and (b) the average numbers of key terms needed in successful trials for different recognition accuracies for the three ranking methods for parts (A)(B)(C)(D) in case (1) and (2), in Table 2.

\section{CONCLUSION}

In this paper we proposed using Reinforcement Learning to rank key terms to guide the user more efficiently. In Reinforcement Learning we proposed using simulated users as training data, and asserted that learnt key term ranking can reflect the semantic structure of the document archive. Extensive simulation results showed that the proposed ranking algorithm offered much better performance than convetnional methods and is relatively robust to recognition errors.

5. REFERENCES

[1] Y.-C. Pan, C.-C Wang, Y.-C Hsieh, T.-H Lee, Y.-S Lee, Y.-S Fu, Y.-T Huang, and L.-S Lee, "A multi-modal dialogue system for information navigation and retrieval across spoken document archives with topic hierarchies," in $A S R U$ 2005, pp. 375-380.

[2] Ya chao Hsieh, Yu tsun Huang, Chien chih Wang, and Lin shan Lee, "Improved spoken document retrieval with dynamic key term lexicon and probabilistic latent semantic analysis (plsa)," in ICASSP, 2006.

[3] Richard S. Sutton and Andrew G. Barto, Reinforcement Learning: An Introduction, MIT Press, 1998.

[4] Marilyn A. Walker, "An application of reinforcement learning to dialogue strategy selection in a spoken dialogue system for email," Journal of Artificial Intelligence Research 12, pp. 387-416, 2000.

[5] Esther Levin, Roberto Pieraccini, and Wieiand Eckert, "Using markov decision process for learning dialogue strategies," in ICASSP, 1998, pp. 201-204.

[6] Andrew G. Barto, Steven J. Bradtke, and Satinder P. Singhand, "Learning to act using real-time dynamic programming," Artificial Intelligence Journal, vol. 72, pp. 81-138, 1995 .

[7] G. W. Furnas, S. Deerwester, S. T. Dumais, T. K. Landauer, R. A. Harshman, L. A. Streeter, and K. E. Lochbaum, "Information retrieval using a singular value decomposition model of latent semantic structure," in SIGIR. 1988, pp. 465-480, ACM Press.

[8] Thomas Hofmann, "Probabilistic latent semantic indexing," in SIGIR, 1999, pp. 50-57.

[9] Y.-C. Pan, Y.-Y. Liu, and L.-S. Lee, "Named entity recognition from spoken documents using global evidences and external knowledge sources with applications on mandarin chinese," in ASRU, 2005, pp. 296-301.

[10] S. E. Robertson, "On term selection for query expansion," Jounal of Documentation, vol. 46, pp. 129-146, 1990. 